\title{
Gestion optimisée de la parenté et de la consanguinité dans les programmes de sélection des bovins laitiers
}

\author{
J.-J. COLLEAU ${ }^{1}$, S. MOUREAUX ${ }^{2}$ \\ ${ }^{1}$ INRA, Station de Génétique Quantitative et Appliquée, F-78352 Jouy-en-Josas Cedex \\ ${ }^{2}$ Institut de l'Elevage, INRA, Station de Génétique Quantitative et Appliquée, F-78352 Jouy-en-Josas Cedex \\ Courrielugencjj@dga2.jouy.inra.fr
}

\begin{abstract}
La sélection intensive chez les animaux domestiques est efficace à court terme mais entraine l'augmentation de la consanguinité, ce qui constitue un handicap pour le long terme. Il est alors essentiel de chercher à définir et à tester en vraie grandeur une méthode de sélection qui réalise un compromis raisonnable entre progrès génétique et augmentation de la consanguinité.
\end{abstract}

La sélection conduit d'une manière générale à privilégier la diffusion de certaines familles présentant une bonne valeur génétique pour 1'objectif de sélection (synthèse sur plusieurs caractères). Les méthodes d'évaluation renforcent d'autant plus le phénomène que l'objectif de sélection comporte des caractères à faible héritabilité (c'est le cas de beaucoup de caractères dits fonctionnels chez les bovins laitiers), parce qu'alors les informations apportées par les performances des apparentés ont beaucoup d'importance dans l'évaluation d'un individu (Verrier et al 1993). Enfin, les méthodes disponibles de reproduction intensive, telles que l'insémination artificielle, constituent à l'évidence un puissant facteur de concentration familiale.

L'efficacité à court terme d'une sélection utilisant à la fois les potentialités de méthodes d'évaluation et de reproduction performantes n'est plus à démontrer. Cependant, il convient de se représenter les conséquences à long terme d'une exploitation intense de la variabilité génétique. D'abord, pour un objectif de sélection stable, le progrès génétique annuel est destiné à se réduire petit à petit, en raison d'une diminution de la variabilité génétique. Celle-ci concerne non seulement les caractères sélectionnés mais aussi les caractères non inclus dans l'objectif de sélection : la population devient donc moins souple vis-à-vis d'un changement d'objectif si les circonstances économiques le demandent. Par ailleurs, alors que certains gènes disparaissent, d'autres sont surreprésentés. On peut ainsi trouver chez un même individu deux copies d'un même gène ancêtre : la probabilité d'un tel évènement connaissant la généalogie complète est le coefficient de consanguinité (noté habituellement F) ou de manière équivalente, le coefficient de parenté entre le père et la mère (Malécot 1948). La consanguinité fait ainsi son apparition et augmente régulièrement, ce qui porte préjudice aux performances des animaux, particulièrement pour les caractères de viabilité, de fertilité et de résistance aux maladies (dépression de consanguinité). Par ailleurs, la consanguinité constitue une sorte de caisse de résonance pour les anomalies héréditaires, notamment pour celles à déterminisme récessif. En effet, pour une fréquence génique donnée de l'anomalie, la fréquence des homozygotes, qui expriment donc l'anomalie, croit avec la consanguinité.

La question concrète qui se pose est ainsi la gestion conjointe du progrès génétique et de la variabilité génétique sur le long terme. L'objectif de ce texte est de présenter, plus en détail que dans Colleau et al (2003), les résultats d'une méthode de gestion optimisée effec- tuée en vraie grandeur sur les populations sélectionnées Holstein, Montbéliarde, Normande (les trois principales races laitières françaises). Dans ces populations, des études descriptives relativement récentes (Boichard et al 1996, 1997, Moureaux et al 2000) ont en effet confirmé à la fois l'étroitesse de la base génétique et l'établissement d'un rythme conséquent d'élévation du niveau de consanguinité (de l'ordre de $+0,2 \%$ par an). Auparavant, on situera la méthode choisie dans son contexte scientifique, puisque cette thématique suscite dans le monde entier de nombreux travaux de génétique quantitative.

\section{1 / Approches de la géné- tique quantitative}

\section{1 / Premières approches}

Schématiquement, entre 1985 et la fin des années 1990, les généticiens quantitatifs ont tenté de résoudre le problème en modifiant les index de sélection de telle sorte que la sélection induise moins de parenté (prélude à la consanguinité). Divers moyens ont été étudiés comme la pénalisation des index par le coefficient de consanguinité de l'individu ou par la parenté avec les futurs conjoints de l'individu (Wray et Goddard 1994, Villanueva et al 
1994, Brisbane et Gibson 1995a, 1995 b) et la diminution de l'importance accordée aux performances des apparentés. Dans ce dernier cas, le résultat recherché pouvait être obtenu de manière très simple en augmentant artificiellement les valeurs d'héritabilité considérées dans l'évaluation (Grundy et Hill 1993, Grundy et al 1994) ou de manière plus complexe, en isolant explicitement dans l'index la part expliquée par les apparentés puis en la sous-pondérant (Toro et PerezEnciso 1990, Verrier et al 1993, Villanueva et al 1994, Wu et Schaeffer 2000). Vu les résultats de simulation, ces solutions ont permis de réduire sensiblement les niveaux de consanguinité et de parenté tout en ne diminuant que modérément les niveaux de progrès génétique. Cependant, une diffusion de tels index modifiés dans le monde des sélectionneurs aurait porté atteinte à la lisibilité des évaluations génétiques parce qu'elles auraient alors perdu leurs propriétés d'indicateurs optimaux des niveaux génétiques.

\section{2 / Approches actuelles}

En quelques mots, les approches actuelles s'effectuent dans le cadre d'une théorie explicite, dûment validée.

L'ensemble des conséquences défavorables de la sélection peut se connecter à un seul indicateur quantitatif : le taux d'augmentation de la consanguinité, noté habituellement $\Delta \mathrm{F}$ (Crow et Kimura 1970, Minvielle 1990, Falconer et MacKay 1996, Ollivier 2002). Il exprime la vitesse relative, par unité de temps, avec laquelle le coefficient de consanguinité $F$ se rapproche de 1. En raisonnant entre les générations $t$ et $t+1$, on a alors

$$
\Delta F=1-\left(1-F_{t+1}\right) /\left(1-F_{t}\right)=\left(F_{t+1}-F_{t}\right) /\left(1-F_{t}\right)
$$

Pour une valeur donnée de $\Delta \mathrm{F}$, l'évolution de F est évidemment curvilinéaire puisque bornée par 1 .

La quantité $\mathrm{N}_{\mathrm{g}}=1 /(2 \Delta \mathrm{F})$ est souvent appelée «effectif génétique». C'est l'effectif d'une population idéalisée produisant le même $\Delta \mathrm{F}$ que la population réelle, de taille plus petite, où les mâles et femelles sont en effectifs égaux et ont chacun même potentiel de reproduction. Dans les populations à structure familiale très simple, le $\Delta \mathrm{F}$ est d'autant plus élevé que la variabilité de la taille des familles est importante (Hill 1972, 1979, Caballero 1994, Santiago et Caballero 1995). Dans le cas de populations quelconques (structure familiale compliquée, chevauchement des générations), Wray et Thompson (1990) ont établi que ce paramètre était d'autant plus élevé que la variabilité des contributions à long terme des reproducteurs d'une génération donnée était grande et ont donné le lien analytique entre les deux (confirmation par Woolliams et Thompson 1994, Woolliams et Bijma 2000). Ce résultat théorique a été mis à profit pour affiner les prédictions de $\Delta \mathrm{F}$ dans des populations simplifiées, sélectionnées classiquement (Woolliams et al 1993, Woolliams et Thompson 1994, Wray et al 1994, Bijma et Woolliams 1999, 2000, Woolliams et Bijma 2000 ) et pour mieux analyser les conséquences de diverses stratégies de sélection.

Les populations réelles présentent des généalogies très complexes et évoluent dynamiquement. On a à tout moment besoin d'un critère permettant d'évaluer les conséquences de décisions de sélection sur l'évolution de F. Le consensus scientifique actuel est que ce critère doit évaluer ces conséquences non à la génération suivante mais environ deux générations plus loin (strictement deux quand les générations sont séparées), ce qui rend plus précise la prédiction de F. Le critère considéré est alors le coefficient de parenté moyen deux à deux entre tous les animaux de la population, en mélangeant tous les sexes et tous les groupes d'âge, assimilant donc la population à un pool génétique unique (Lacy 1995, Caballero et Toro 2000). Cela implique de tenir compte non seulement des parentés entre mâles et femelles mais aussi des parentés entre individus de même sexe, ce qui n'est pas aussi paradoxal qu'il pourrait le sembler. Considérons en effet les parentés entre deux mâles contemporains, A et B : dans le pedigree d'un individu né plusieurs générations plus tard (dès la génération d'après si les générations ne sont pas chevauchantes), on pourra en effet trouver $A$ dans la généalogie paternelle et $B$ dans la généalogie maternelle. Même raisonnement pour des reproducteurs situés dans des groupes d'âge différent. Il faut ajouter que le coefficient de parenté moyen évoqué plus haut tient compte des taux d'utilisation instantanés des reproducteurs : il s'agit donc d'un taux d'utilisation moyen pondéré. Ce point est également logique : dans l'exemple précédent, plus A est utilisé, plus on a de chances de le retrouver à la fois dans la généalogie paternelle et dans la généalogie maternelle, plusieurs générations plus tard.
Ballou et Lacy (1995) ont vérifié par simulation qu'en minimisant le critère de parenté moyenne lors de la mise en reproduction, on préserve le mieux la variabilité génétique issue de la population ancestrale (maximisation du nombre d'allèles ancestraux encore présents). Par ailleurs, la théorie de la prédiction du $\Delta \mathrm{F}$ à partir du coefficient de parenté moyen pondéré (Meuwissen 1997, Caballero et Toro 2000) a pu être validée en la confrontant aux résultats obtenus par simulation sur des populations sélectionnées simplifiées : le $\Delta \mathrm{F}$ trouvé expérimentalement est bien celui qui était prévu lors de la sélection des reproducteurs et qui était intégré comme contrainte dans les équations d'optimisation (Sonesson et Meuwissen 2000, 2002).

Dès lors, toute la question de la gestion combinée du progrès génétique se résume à la détermination de la liste des reproducteurs sélectionnés avec leurs taux d'utilisation calculés «au mieux» (Woolliams 1998, Woolliams et al 2002). Dans la très grande majorité des travaux actuels (Meuwissen 1997, Meuwissen et Sonesson 1998, 2000, 2002, Sonesson et al 2000), on se donne un $\Delta \mathrm{F}$ considéré a priori comme raisonnable (la plupart du temps, $1 \%$ par génération). Sous cette contrainte, et compte tenu de la structure généalogique présente de la population, on calcule la liste des reproducteurs et les taux d'utilisation qui maximisent le progrès génétique. Les simulations ont montré que les populations sélectionnées suivant ce principe étaient capables de générer 20 à $30 \%$ de progrès génétique de plus que des populations de plus grande taille, sélectionnées «classiquement» (sélection avec troncature puis utilisation uniforme des reproducteurs) et produisant le même $\Delta \mathrm{F}$. On pouvait avoir un doute sur le réalisme des résultats obtenus pour la sélection dite classique, étant donné que les sélectionneurs tiennent compte de la parenté dans une certaine mesure et en revanche sont loin de pratiquer une utilisation uniforme des reproducteurs. Ce doute est levé depuis les travaux de Avendano et al (2003) et Kearney et al (2004) qui ont confirmé ces ordres de grandeur sur populations réelles.

\section{2 / Principes des tests sur les populations françaises}

\section{1 / Contrainte principale}

On considère que les sélectionneurs sont plus familiarisés avec les niveaux 
de progrès génétique qu'avec les niveaux de consanguinité et de parenté. On leur demande donc non pas de fixer la valeur de $\Delta \mathrm{F}$ mais de donner simplement la valeur du progrès génétique qu'ils souhaitent. Cette idée a déjà été évoquée de manière indirecte par Toro et Nieto (1984). La tâche de l'optimisation est alors de définir les choix qui conduiront à l'établissement d'un coefficient de parenté le plus bas possible, sous la contrainte du progrès génétique souhaité.

\section{2 / Identification des étapes}

Il s'agit de bien situer les étapes de sélection où des décisions sont prises et où le double éclairage, sous l'angle des niveaux génétiques et sous l'angle de la variabilité génétique, est nécessaire. On en a identifié quatre, qui ont été testées en vraie grandeur. Chaque phase peut être traitée en tenant compte de contraintes spécifiques qui s'ajoutent à la contrainte principale.

\section{Les quatre étapes sont les suivantes :}

Amont : il s'agit de la procréation des jeunes taureaux à partir de pères et de mères à taureaux. Ceux-ci suivent ensuite les étapes habituelles de la sélection jusqu'à la mise en testage sur descendance. Le terme amont n'est pas courant mais est utilisé ici pour éviter les périphrases.

Mise en testage : il s'agit du choix vers 10 mois des jeunes taureaux pour les prochaines étapes de sélection du schéma, c'est-à-dire essentiellement la mise en testage et secondairement la fonction sexuelle.

Agrément : dans le cadre de la Loi sur l'Elevage de 1966, un taureau ne peut être utilisé pour l'insémination artificielle que s'il est agréé, au vu de ses aptitudes à améliorer le niveau génétique de la population pour toute une série de caractères économiquement importants. Il s'agit donc ici de faire la sélection parmi les nouveaux taureaux qui viennent d'être testés.

Aval : il s'agit ici de déterminer les taux d'utilisation des taureaux mis en service. Dans la version "aval détaillé», on désigne en plus les vaches sur lesquelles les taureaux doivent être utilisés (détermination des accouplements).

\section{3 / Test a posteriori}

On considère des données réelles récentes, correspondant à chacune des quatre étapes et l'on cherche à optimiser rétrospectivement les décisions, compte tenu de la situation de l'époque et en se donnant comme contrainte principale le niveau génétique moyen correspondant aux décisions réellement prises. En d'autres termes, on cherche à répondre avec précision à la question : aurait-il été possible de réduire significativement les parentés sans aucune perte de progrès génétique?

\section{3 / Phase amont}

\section{1 / Objectif et contraintes}

L'objectif est ici de minimiser la parenté moyenne dans une population de taureaux non encore testés sur descendance mais qui auront une incidence pour l'avenir. Cette population de taureaux mélange les séries annuelles de taureaux en cours de testage (en général 4) et la série des jeunes taureaux qu'on doit procréer à l'issue d'accouplements entre pères à taureaux (PAT) et mères à taureaux (MAT). On gère donc la variabilité non seulement instantanément mais aussi dans le temps. Dans ce contexte, les reproducteurs déjà très utilisés sont logiquement pénalisés.

On considère ensuite que la sélection sur les caractères économiquement importants s'exerce uniquement au travers de la sélection sur un index de synthèse, car c'est la manière la plus efficace de procéder pour créer des progrès génétiques sur un ensemble de caractères. L'index de synthèse pour les bovins laitiers en France est l'ISU : il a été redéfini récemment (Colleau et Regaldo 2001) et donne autant d'importance aux caractères fonctionnels qu'aux caractères laitiers. On demande alors que le niveau moyen ISU des produits des accouplements, estimé sur ascendance, soit égal à une certaine valeur désirée (le niveau réel observé dans le cas du test rétrospectif).

Par ailleurs, la méthode de reproduction utilisée pour les mères à taureaux peut impliquer la superovulation suivie de transfert embryonnaire (notée TE), qui est une technique coûteuse. De ce fait, on ajoute une contrainte supplémentaire : le coût de la campagne de reproduction des MAT (pour le test rétrospectif, il est égal au coût réel correspondant). Sans cette contrainte, on a observé que l'optimisation conduit alors à utiliser massivement la TE pour un nombre plus réduit de vaches, mais induit un surcoût total notable, ce qui est à éviter. Pour traiter correctement le problème, la méthode de calcul utilise les renseignements détaillés fournis par le sélectionneur. Celui-ci définit les différents niveaux de reproduction possibles. Par exemple, pour le test rétrospectif, on a considéré 3 niveaux : une seule insémination artificielle (IA) ou une TE suivie d'une IA ou deux TE suivies d'une IA. Pour chacune des éventualités, le nombre moyen et le coût attendus des veaux mâles sont évidemment précisés.

\section{2 / Méthode de calcul}

On détermine d'abord le nombre et l'identité des femelles affectées à chaque niveau de reproduction. Ensuite, on détermine les accouplements à effectuer à chaque action élémentaire de reproduction (TE ou IA).

A la base, on dispose d'une large liste de PAT et de MAT disponibles (les «candidats » et «candidates»), fournie par le sélectionneur, et l'on considère la totalité des accouplements potentiels entre ces candidats et candidates. On cherche alors à déterminer la fréquence optimale de chacun de ces accouplements, connaissant les contraintes. Les solutions sont données par la résolution d'un système linéaire de très grande taille impliquant de nombreux termes non nuls correspondant aux coefficients de parentés entre des individus futurs issus d'accouplements différents. Il peut couramment y avoir des milliards de termes car leur nombre est égal au carré du produit du nombre de candidats par le nombre de candidates. Les calculs sont néanmoins réalisables si l'on utilise une méthode indirecte de calcul des coefficients de parenté, rapide et économe en utilisation de mémoire d'ordinateur, qui exploite la structure particulière de l'inverse de la matrice de parenté (Colleau 2002, Sargolzaei et al 2005a). L'optimisation analytique dans ce cadre est relativement simple. On examine les performances d'un jeu donné de fréquences en terme de parenté moyenne 2 à 2 (dans la population des futurs individus et des taureaux en cours de testage) et en terme d'index moyen sur ascendance pour l'ISU. Puis au moyen de méthodes spécialisées, on change le jeu de fréquences pour avoir un résultat plus satisfaisant et ainsi de suite. Cependant, la solution obtenue n'est pas encore réaliste. Par exemple, une vache qui doit être collectée une fois puis inséminée peut se voir attribuer simultanément 5 taureaux avec des fréquences différentes alors qu'au maximum 2 taureaux peuvent être impliqués (un à chaque opération élémentaire). La phase finale de l'optimisation consiste alors à réaliser itérativement la double opération suivante : 
abandon très progressif (par exemple $5 \%$ des accouplements encore en jeu) des accouplements les moins recommandés, puis nouvelle optimisation concernant les accouplements restants. A la fin des calculs, on peut ainsi déterminer le taureau optimal pour chaque vache à chacune des opérations élémentaires. Le détail de tous les algorithmes de calcul utilisés est donné dans Colleau et al (2004a).

\section{3 / Données considérées}

Les trois grandes races laitières exploitées en France sont représentées. Les données des races Holstein $(\mathrm{PH})$, Montbéliarde (MO) et Normande (NO) proviennent respectivement des programmes de sélection conduits par les organismes de sélection Oger, Umotest et Gna. La population Normande est échantillonnée en totalité puisque le Gna est l'organisme qui coordonne les opérations d'amont dans cette race. On considère les veaux mâles entrés en station de performance-test entre le $1^{\text {er }}$ mars 2002 et le 28 février 2003, au nombre de 499, 359 et 401 dans l'ordre $\mathrm{PH}, \mathrm{MO}, \mathrm{NO}$. On considère également 4 séries de taureaux en cours de testage soit 585, 708 et 626 individus respectivement.

\section{4 / Résultats}

Le tableau 1 montre les résultats obtenus. La parenté moyenne dans les séries en cours de testage est de l'ordre de $6 \%$. Pour fixer les idées, tout se passe comme si deux individus pris au hasard dans ce groupe avaient deux grands-parents en commun. Les parentés observées dans la série réelle en sta-

Tableau 1. Amont : parentés concernant les jeunes taureaux en station et les taureaux déjà en testage.

\begin{tabular}{|c|c|c|c|}
\hline $\begin{array}{c}\text { Parentés } \\
\text { Moy. 2 à 2 } \\
\text { (\%) }\end{array}$ & Réel & Opt. & $\begin{array}{c}\text { Réduction } \\
\text { (\%) }\end{array}$ \\
\hline Testage & 6,47 & & \\
& 5,88 & $i d$. & 0 \\
\hline Testage vs & 6,07 & & \\
Station & 5,44 & 3,82 & 25 \\
\hline Station & 5,87 & 4,13 & 28 \\
\hline Testage + & 5,76 & 6,05 & 22 \\
Station & 6,07 & 5,54 & 24 \\
\hline Consanguinité & 5,84 & 5,10 & 27 \\
en station & 4,20 & 2,967 & 14 \\
(\%) & 4,18 & 3,15 & 16 \\
\hline
\end{tabular}

Verticalement : Holstein, Montbéliard,

Normand. tion sont du même ordre. Si cette série de jeunes taureaux avait pu être obtenue après optimisation, on aurait pu diminuer substantiellement les parentés (de 22 à $27 \%$ suivant les races).

La parenté moyenne entre les jeunes taureaux réels et les séries en testage est de l'ordre de 5,5\%. Après optimisation, on aurait pu également la faire baisser de façon conséquente : de 25 à $30 \%$ au total. Donc, l'optimisation aurait apporté davantage de diversité tant intra-année qu'entre années. Au total, la réduction de parenté moyenne est plus faible (de 14 à $18 \%$ ) que les valeurs citées plus haut, en raison du poids des séries en testage (elles ne dépendent pas de l'optimisation, puisqu'elles sont déjà connues). Bien que 1'optimisation vise à minimiser la consanguinité à long terme, on peut constater qu'elle est déjà efficace à court terme puisqu'elle permettrait de faire baisser la consanguinité des jeunes taureaux de 22 à $29 \%$.

\section{4 / Phase mise en testage}

\section{1 / Objectif et contraintes}

L'objectif est ici aussi de minimiser la parenté moyenne dans une population de taureaux non encore testés sur descendance. On considère toujours les séries annuelles de taureaux en cours de testage, mais cette fois, on y ajoute la série de jeunes taureaux nouvellement mis en testage, qui sont sélectionnés dans l'ensemble des $\mathrm{N}$ taureaux produits par la phase amont. Il y a alors deux contraintes à respecter : le nombre $n$ de jeunes taureaux sélectionnés pour la mise en testage et leur niveau moyen pour un index de synthèse.

\section{2 / Méthodes de calcul}

Elles sont beaucoup plus simples que pour la phase amont. En résolvant des systèmes linéaires de taille très raisonnable (quelques centaines d'inconnues), on construit progressivement un classement complet des individus qui sortent de la station et qui sont candidats à la mise au testage. On repère d'abord les meilleurs et les moins bons, et ainsi de suite en progressant vers le milieu du classement. Sur cette liste ordonnée, les $n$ premiers sont les individus à mettre en testage.

\section{3 / Données considérées}

Il s'agit des candidats nés entre le 1 er juillet 2002 et le 30 juin 2003. Les mâles déjà en testage sont nés entre le $1^{\text {er }}$ juillet 2000 et le 30 juin 2002. Ces taureaux présentent tous la particularité d'être impliqués dans le programme expérimental de Sélection Assistée par Marqueurs (SAM), qui a précisément pour but d'affiner les choix lors de mise au testage, grâce à l'utilisation de marqueurs moléculaires proches de gènes en ségrégation ayant une incidence sur les performances, les QTLs (Quantitative Trait Loci). Les principes et potentialités de la SAM ont été déjà décrits à plusieurs occasions (Colleau 1999, Fritz et al 2003). La SAM concerne les taureaux des races $\mathrm{PH}$, MO, NO, issus de 8 programmes de sélection autonomes et fait 1'objet d'une expérimentation pilote impliquant l'UNCEIA, l'INRA et Labogena (laboratoire de génotypage moléculaire). L'évaluation génétique de ces taureaux fournit un index de synthèse nommé ISS, très proche de l'ISU.

Le marquage moléculaire offre la possibilité de calculer des parentés au niveau de chaque QTL (Sargolzaei et al 2005b). Ces parentés sont différentes des parentés classiques, qui forment un continuum, parce qu'on peut mieux suivre la transmission effective des gènes. De ce fait, on peut mieux cerner dans quelle situation on se trouve. En effet, il n'y a que deux possibilités : les deux individus considérés portent ou ne portent pas la copie d'un même gène ancêtre. Les parentés entre deux individus donnés peuvent être extrêmement différentes suivant le QTL considéré. $\mathrm{Vu}$ la multiplicité des QTLs impliqués, il n'est donc pas étonnant de constater, à l'issue d'un calcul complet, que la parenté moyenne pour tous les QTLs est très proche de la parenté classique. Pour cette raison, on continue de considérer celle-ci dans la méthode d'optimisation.

Les effectifs engagés dans la comparaison sont indiqués au tableau 2.

Tableau 2. Effectifs correspondant à la mise en testage des jeunes taureaux.

\begin{tabular}{|c|c|c|c|c|}
\hline \multirow{2}{*}{ Race } & $\begin{array}{c}\text { Unité } \\
\text { de sélection }\end{array}$ & T & C & S \\
\hline \multirow{4}{*}{ PH } & Gdh & 337 & 248 & 123 \\
\cline { 2 - 5 } & Midatest & 337 & 247 & 50 \\
\cline { 2 - 5 } & Oger & 731 & 415 & 93 \\
\cline { 2 - 5 } & Uneco & 756 & 554 & 212 \\
\cline { 2 - 5 } & Urceo & 727 & 647 & 117 \\
\hline MO & Umotest & 587 & 376 & 130 \\
\hline NO & Gna & 751 & 396 & 146 \\
\hline
\end{tabular}

$\mathrm{T}=$ déjà en testage $; \mathrm{C}=$ candidats ;

$\mathrm{S}=$ Sélectionnés 


\section{4 / Résultats}

Les résultats obtenus figurent aux tableaux 3 et 4 . Ils indiquent que l'optimisation permet comme dans la phase amont de diminuer la parenté tant à l'intérieur de la nouvelle série sélectionnée qu'entre celle-ci et les anciennes séries. L'ordre de grandeur de la réduction est de $15 \%$ (de 11 à $20 \%$ suivant les programmes de sélection particuliers).

Tableau 3. Mise en testage des jeunes taureaux. Coefficients de parenté (\%) entre les sélectionnés.

\begin{tabular}{|c|c|c|c|c|}
\hline Race & $\begin{array}{c}\text { Unité de } \\
\text { sélection }\end{array}$ & Réels & Opt. & $\begin{array}{c}\text { Réduction } \\
(\%)\end{array}$ \\
\hline \multirow{4}{*}{ PH } & Gdh & 6,4 & 5,7 & 11 \\
\cline { 2 - 5 } & Midatest & 6,9 & 5,7 & 17 \\
\cline { 2 - 5 } & Oger & 6,2 & 5,4 & 14 \\
\cline { 2 - 5 } & Uneco & 5,7 & 4,8 & 17 \\
\cline { 2 - 5 } & Urceo & 5,7 & 4,6 & 20 \\
\hline MO & Umotest & 6,4 & 5,4 & 16 \\
\hline NO & Gna & 6,8 & 5,8 & 15 \\
\hline
\end{tabular}

Tableau 4. Mise en testage des jeunes taureaux. Coefficients de parenté (\%) entre les taureaux déjà en testage et les sélectionnés.

\begin{tabular}{|c|c|c|c|c|}
\hline Race & $\begin{array}{c}\text { Unité de } \\
\text { sélection }\end{array}$ & Réels & Opt. & $\begin{array}{c}\text { Réduction } \\
\text { (\%) }\end{array}$ \\
\hline \multirow{4}{*}{ PH } & Gdh & 5,8 & 5,3 & 10 \\
\cline { 2 - 5 } & Midatest & 5,4 & 4,5 & 16 \\
\cline { 2 - 5 } & Oger & 5,5 & 4,6 & 15 \\
\cline { 2 - 5 } & Uneco & 5,3 & 4,5 & 14 \\
\cline { 2 - 5 } & Urceo & 5,4 & 4,4 & 19 \\
\hline MO & Umotest & 5,4 & 4,4 & 19 \\
\hline NO & Gna & 5,1 & 4,5 & 11 \\
\hline
\end{tabular}

\section{5 / Phase aval}

La phase agrément se place chronologiquement avant la phase aval. Cependant, il est plus facile d'exposer la méthode d'optimisation de la phase agrément après celle de la phase aval, parce que la première est une adaptation de la seconde.

\section{1 / Objectif et contraintes}

L'objectif est de minimiser la parenté moyenne dans une population intégrant les femelles existantes de tout âge et les produits femelles attendus de la campagne de reproduction envisagée, impliquant les femelles en âge d'être inséminées (liste non sujette à optimisation) et les taureaux agréés disponibles (candidats). La contrainte est que le niveau moyen ISU des taureaux après pondération par le taux d'utilisation soit égal à une valeur désirée.

\section{2 / Méthode de calcul}

On considère la totalité des accouplements potentiels entre les candidats et les femelles à inséminer. Pour limiter le volume des calculs, on simplifie les pedigrees en séparant les femelles en groupes d'animaux ayant le même père (P) et le même grand-père maternel (GPM). On cherche alors à déterminer la fréquence optimale de chacun de ces «accouplements» (groupe PGPM* taureau d'IA), connaissant la contrainte. L'algorithme de calcul rappelle beaucoup celui utilisé pour la phase amont. In fine, on peut déduire les taux d'utilisation des taureaux pour chaque groupe et les taux globaux.

\section{3 / Données considérées}

On prend en compte les inséminations premières réalisées entre le $1^{\mathrm{er}}$ octobre 2001 et le 30 septembre 2002. Les vaches sélectionnées, à généalogie connue, sont au nombre de 219169, 124937, 135771 pour les races PH (Oger), MO (Umotest), NO (Gna). Les nombres respectifs de groupes PGPM sont de 1875,1861 et 2075. Les nombres de taureaux agréés utilisés sont respectivement de 50, 52 et 42 .

\section{4 / Résultats}

Comme pour la partie amont, la procédure d'optimisation permet de réduire sensiblement les parentés entre les animaux nouvellement produits (de 12 à $19 \%$ ) et entre ceux-ci et les animaux déjà existants (de 19 à $29 \%$ ). La comparaison des taux globaux recommandés d'utilisation et des taux réels montre qu'il n'y a aucune corrélation entre les deux, ce qui souligne la marge de progrès qu'il y aurait à réaliser, si l'on désirait vraiment canaliser la demande des éleveurs.

Tableau 5. Aval simplifié (groupes $\left.P^{\star} G P M\right)$. Coefficients de parenté concernant les femelles.

\begin{tabular}{|c|c|c|c|}
\hline $\begin{array}{c}\text { Parentés } \\
(\%)\end{array}$ & Réel & Opt. & $\begin{array}{c}\text { Réduction } \\
(\%)\end{array}$ \\
\hline Existantes & $\begin{array}{l}6,86 \\
6,76 \\
6,20\end{array}$ & id. & 0 \\
\hline $\begin{array}{l}\text { Existantes vs } \\
\text { nouvelles }\end{array}$ & $\begin{array}{l}4,11 \\
4,06 \\
3,82\end{array}$ & $\begin{array}{l}3,61 \\
3,27 \\
3,30\end{array}$ & $\begin{array}{l}12 \\
19 \\
14\end{array}$ \\
\hline Nouvelles & $\begin{array}{l}5,52 \\
5,47 \\
5,20\end{array}$ & $\begin{array}{l}4,45 \\
3,86 \\
4,08\end{array}$ & $\begin{array}{l}19 \\
29 \\
21\end{array}$ \\
\hline $\begin{array}{c}\text { Existantes + } \\
\text { nouvelles }\end{array}$ & $\begin{array}{l}6,27 \\
6,18 \\
5,60\end{array}$ & $\begin{array}{l}6,14 \\
6,00 \\
5,46\end{array}$ & $\begin{array}{l}2 \\
3 \\
3\end{array}$ \\
\hline
\end{tabular}

Verticalement : Holstein, Montbéliard, Normand.

\section{6 / Phase agrément}

La méthode d'optimisation de la phase agrément est très semblable à celle de la phase aval. Les femelles considérées et leurs groupes sont les mêmes. La seule différence est qu'on met en compétition les taureaux déjà agréés et les taureaux nouvellement testés. A l'issue du calcul, les jeunes taureaux qui ne figurent pas sur la liste des taureaux d'utilisation recommandée ne peuvent prétendre à l'agrément.

\section{1 / Données considérées}

On considère les taureaux proposés à l'agrément en juillet 2002, soit 208, 130 et 152 taureaux pour la race $\mathrm{PH}$ (Oger), MO (Umotest), NO (Gna) respectivement. Les nombres respectifs de taureaux effectivement agréés sont de 32,15 et 19 , ce qui correspond aux très fortes pressions de sélection habituellement rencontrées dans cette phase (environ $90 \%$ des taureaux sont éliminés!).

\section{2 / Résultats}

On constate au tableau 6 que l'agrément aurait dû être refusé à plus de la moitié des taureaux officiellement agréés, non pas à cause d'un ISU insuffisant mais d'une trop forte parenté avec des familles déjà très représentées dans la population. Inversement, il aurait convenu d'agréer des taureaux à index ISU proche de la limite actuelle d'exclusion mais moins apparentés à la population. Ceux-ci constituent environ le $1 / 3(15 / 41$ pour l'ensemble des trois races) des agréments optimisés. Pour l'avenir, c'est vers ceux-ci que devrait porter l'effort des organismes de sélection. Normalement, de tels taureaux seraient automatiquement sélectionnés par les procédures d'optimisation pour l'aval détaillé ( $c f$ plus loin). On remarquera que par rapport à la réalité, l'optimisation donne l'agrément à moins de taureaux, mieux ciblés. Cela illustre bien le fait que la bonne gestion de la variabilité génétique n'est pas uniquement une question d'effectifs.

Tableau 6. Agrément des taureaux.

\begin{tabular}{|c|c|c|c|}
\hline Race & A+ & A- & R+ \\
\hline PH & 10 & 22 & 6 \\
\hline MO & 6 & 9 & 7 \\
\hline NO & 10 & 9 & 2 \\
\hline
\end{tabular}

A+ : agréé en réel et en optimisé,

A- : agréé en réel mais pas en optimisé,

$\mathrm{R}+$ : refusé en réel mais agréé en optimisé. 


\section{7 / Phase aval détaillé}

La phase aval décrite précédemment suppose que la sélection sur les caractères économiquement intéressants s'exerce uniquement au travers de l'ISU. Ce mode de gestion n'est pas en fait rigoureusement appliqué, notamment au niveau des éleveurs de base. Très souvent, ils sélectionnent les mâles d'IA qu'ils utilisent non seulement en fonction du niveau génétique de synthèse mais aussi en fonction de qualités particulières, complémentaires de défauts correspondants chez la vache à inséminer : les accouplements ont alors une fonction correctrice marquée. Il convient d'être réaliste, en admettant que les éleveurs sont très attachés à cette idée et donc en modifiant en conséquence les méthodes de gestion de la variabilité génétique pour la phase aval. L'idée directrice est qu'en satisfaisant au mieux les éleveurs dans le détail des accouplements, on augmente les chances de maîtriser réellement les taux d'utilisation des taureaux, qui sont les paramètres essentiels en matière de variabilité génétique. D'où les développements qui suivent.

\section{1 / Objectif et contraintes}

Il faut d'abord satisfaire l'intérêt général. Il s'agit donc de minimiser la parenté moyenne dans la population des femelles existantes et des femelles issues des accouplements, comme dans la phase aval précédemment décrite, tout en respectant une contrainte ISU pour le niveau moyen pondéré des taureaux utilisés. Il s'agit aussi de produire le moins de consanguinité possible avec les accouplements recommandés. Cette dernière clause amène à considérer les pedigrees complets et non simplifiés comme dans l'optimisation de la phase aval.

Il faut ensuite satisfaire les souhaits particuliers des éleveurs concernant les «corrections» précédemment évoquées.

\section{2 / Méthodes de calcul}

On détermine d'abord le taux d'utilisation optimal (nombre de vaches allouées) des taureaux disponibles en service en respectant la contrainte ISU pour les taureaux sélectionnés.

On définit ensuite une valeur de synthèse pour chaque accouplement. Le problème est alors de maximiser la valeur de synthèse moyenne pour les accouplements retenus, tout en respec- tant la contrainte que le nombre d'accouplements impliquant un taureau donné soit égal au nombre de vaches donné dans la première étape.

Il peut y avoir une infinité de fonctions de synthèse. Ce qui a été testé dans l'exemple numérique des paragraphes 7.3 et 7.4 est une fonction linéaire. La Valeur de Synthèse d'un Accouplement particulier est notée VSA : elle est caractéristique de l'association correspondante taureauvache alors que 1' ISU est caractéristique du seul taureau. On pose alors $\mathrm{VSA}=f \mathrm{VCA}+(1-f)$ ISUA où $f$ est un coefficient situé entre 0 et 1 . $\mathrm{Au}$ début de la procédure de calcul, tous les accouplements possibles entre les vaches et les taureaux sélectionnés se voient attribuer une VSA spécifique.

VCA est la valeur de l'accouplement considéré pour le coefficient de consanguinité. On a alors $\mathrm{VCA}=-\left(\mathrm{F}-\mathrm{F}_{\mathrm{m}}\right) / \sigma_{\mathrm{F}}$ où $\mathrm{F}$ est la consanguinité du produit issu de l'accouplement, $\mathrm{F}_{\mathrm{m}}$ est le $\mathrm{F}$ moyen pour tous les accouplements possibles et où $\sigma_{F}$ est l'écarttype correspondant. Le coefficient négatif résulte du fait qu'on cherche à minimiser le $\mathrm{F}$ des accouplements retenus.

L'ISUA est une modification de l'ISU du taureau impliqué dans l'accouplement en fonction des exigences formulées pour la vache impliquée dans l'accouplement. Les index du taureau pour certains caractères particuliers se voient alors accorder une pondération plus forte que dans l'ISU. En d'autres termes, l'ISUA entérine le mode de pensée de l'éleveur, pour qui le classement des taureaux dépend de la vache considérée. Une fois ces changements de pondérations effectués, l'ISUA est ensuite standardisé (centrage puis division par l'écart-type, évalué sur l'ensemble des taureaux sélectionnés). Après cette standardisation, les ISUA impliquant une vache donnée ont pour moyenne 0 et écart-type 1 , ceci quelle que soit la vache.

Pour un $f$ donné, les accouplements retenus sont établis progressivement, en résolvant un système linéaire d'équations d'optimisation. Les calculs étant très rapides, il est possible de tester toute une série de valeurs de $f$ et de choisir une valeur qui corresponde à un compromis acceptable pour le sélectionneur sous le double aspect de la consanguinité et de l'adéquation zootechnique.

\section{3 / Données considérées}

Elles proviennent de 8897 vaches Normande à généalogie connue, sur la zone d'activité de l'Isno, coopérative de production de semences et bénéficiant d'un service d'accouplements programmés proposé par cet organisme (campagne 2003). Ce service tient compte des souhaits exprimés par les éleveurs (notamment en matière d'index 'morphologie' des taureaux), en ce qui concerne chacune de leurs vaches.

En accord avec cet organisme, la fonction définissant l'ISUA est déterminée de la manière suivante :

Si exigence pour 1 caractère

$\mathrm{ISUA}=0,70 \mathrm{ISU}+0,3$ index caractère 1

Si exigence pour 2 caractères ISUA $=0,50 \mathrm{ISU}+0,3$ index caractère 1 $+0,2$ index caractère 2

\section{Si exigence pour 3 caractères}

ISUA $=0,50$ ISU $+0,25$ index caractère 1 $+0,15$ index caractère $2+0,10$ index caractère 3

Bien évidemment, la définition de la VSA est subjective et a pour but d'essayer de mimer la méthode de jugement pratiquée par les éleveurs. Rappelons que sa définition n'a aucun effet sur les taux d'utilisation des taureaux retenus.

\section{4 / Résultats}

La première phase retient 27 taureaux parmi les 38 possibles et aboutit à réduire de $13 \%$ la parenté moyenne dans l'ensemble des vaches et des taureaux retenus. Le tableau 7 indique les effets d'une variation du coefficient $f$ sur la valeur moyenne des accouplements recommandés, à la fois pour $\mathrm{F}$ et VSA. A la lecture de ce tableau, on pourrait recommander la prise en considération d'un coefficient de

Tableau 7. Aval détaillé : effet de l'importance donnée à la consanguinité.

\begin{tabular}{|c|c|c|}
\hline $\boldsymbol{f}$ & $\begin{array}{c}\text { ISUA } \\
\text { moy }\end{array}$ & $\begin{array}{c}\mathbf{F} \\
\text { moy }\end{array}$ \\
\hline 0 & 1,13 & 4,27 \\
\hline 0,2 & 1,10 & 3,22 \\
\hline 0,4 & 1,05 & 2,86 \\
\hline 0,5 & 1,02 & 2,74 \\
\hline 0,6 & 0,97 & 2,63 \\
\hline 0,8 & 0,82 & 2,46 \\
\hline 1 & 0,29 & 2,36 \\
\hline réel & 0,77 & 3,70 \\
\hline
\end{tabular}

ISUA : synthèse de I'ISU et de caractères spécifiques.

$f$ : poids de la consanguinité dans l'ISA. 
l'ordre de 0,5 qui assure à la fois une réduction notable de $\mathrm{F}$ (de $26 \%$ ) et une meilleure satisfaction des souhaits sur les critères zootechniques, représentés par l'ISUA (+ $32 \%)$.

Il ne s'agit bien entendu que d'une moyenne. Le tableau 8 montre qu'en réalité cette double amélioration par rapport au réel s'est effectuée dans $49 \%$ des cas. Dans $44 \%$ des cas, il y a eu amélioration pour l'un des deux aspects mais détérioration pour l'autre. Dans seulement $7 \%$ des cas, la procédure optimisée a été certainement moins bonne que le choix effectif de l'éleveur.

Tableau 8. Aval détaillé : situation des accouplements optimisés par rapport aux accouplements réels (poids de la consanguinité dans la VSA $=0,5$ ).

\begin{tabular}{|c|c|c|}
\hline & $\begin{array}{c}\text { ISUA } \\
\text { diminue (\%) }\end{array}$ & $\begin{array}{c}\text { ISUA } \\
\text { augmente (\%) }\end{array}$ \\
\hline F diminue & 30 & 49 \\
\hline F augmente & 7 & 14 \\
\hline
\end{tabular}

\section{5 / Extensions prévues}

L'approche devra être affinée pour tenir compte de contraintes supplémentaires. Notamment, l'accessibilité des taureaux d'IA dépend du degré d'implication de l'éleveur en fonction du schéma de sélection. La méthodologie utilisée dans la phase 2 de l'optimisation devrait pouvoir intégrer sans difficulté cette contrainte supplémentaire, si l'information correspondante est donnée de manière exhaustive.

\section{8 / De l'utilité d'un ISU pénalisé pour la parenté (ISUP)}

\section{1 / Perspective}

Les sélectionneurs ont tendance à croire que les problèmes de gestion de la variabilité génétique seront beaucoup mieux appréhendés s'ils choisissent leurs reproducteurs d'après un nouvel index de synthèse. On pourrait l'appeler ISUP pour une bonne lisibilité : c'est l'ISU actuel pénalisé pour la parenté du reproducteur avec la population. Cependant, il a été clairement établi dans la littérature scientifique (cf plus haut) que la manière idéale de procéder consiste à sélectionner en se conformant à des taux d'utilisation optima des reproducteurs, qui sont donnés non par de tels index synthétiques mais par des calculs spécialisés tenant compte de la structure précise des populations (cf plus haut). Ils permettent d'obtenir le meilleur compromis entre les nécessités contradictoires de progrès génétique et de gestion durable de la variabilité génétique.

Les nécessités opérationnelles sont telles que la question d'un éventuel ISUP se pose néanmoins. On a en effet besoin d'un index synthétique simple permettant de comprendre avec une bonne approximation le mérite global d'un individu tel qu'il apparaît à l'issue des procédures d'optimisation. Elles livrent parfois des résultats qui peuvent surprendre. Par exemple, le meilleur taureau en ISU peut être recommandé pour la phase aval mais éliminé de la liste des pères à taureaux. L'introduction du nouvel ISU en 2001 a permis d'affiner notre perception de l'intérêt réel des reproducteurs dans la population par la prise en considération des caractères fonctionnels, qui ont un impact de moins en moins négligeable sur l'économie des exploitations laitières. Au travers de l'ISUP, on effectuerait un classement encore plus réaliste, intégrant l'impact potentiel du reproducteur sur la variabilité génétique de la population et donc le devenir de celle-ci, s'il venait à être effectivement utilisé. Cependant, rappelons-le, établir la hiérarchie des reproducteurs ne donne pas d'indication claire sur le taux d'utilisation. Le premier de la hiérarchie doit-il être impliqué dans $15 \%$ des accouplements ou seulement dans $5 \%$ ? C'est aussi une question opérationnelle, à laquelle seuls les calculs d'optimisation peuvent répondre.

Jusqu'à présent, dans la phase d'optimisation amont, les candidats pères à taureaux et les candidates mères à taureaux sont essentiellement présélectionnés sur l'ISU. L'utilisation d'un ISUP lors de cette présélection pourrait en améliorer la qualité, en donnant une meilleure garantie de ne pas avoir exclu d'office des individus potentiellement intéressants.

En ce qui concerne l'agrément, les sélectionneurs disposent actuellement des deux éléments essentiels que sont l'ISU et la parenté mais n'ont pas d'indication claire sur leur pondération relative. L'établissement d'un ISUP permettrait donc tout naturellement d'éclairer les décisions à effectuer.

La question centrale concernant l'ISUP est la pondération à accorder au critère de parenté. On pourrait considérer l'impact économique de la parenté sur les performances via la dépression de consanguinité mais ce serait très restrictif (voir l'introduction). Il est préférable de viser la préservation maximale de la variabilité génétique, en considérant une pondération d'opportunité : la pondération maximale qu'on peut se permettre de donner au critère de parenté, sans perdre de progrès sur I'ISU. En contrepartie, la sélection sur les caractères économiquement importants est supposée s'exercer uniquement d'après l'ISU, sans sélection complémentaire sur certaines composantes de l'ISU. La méthode d'obtention de cette pondération est décrite en annexe.

\section{2 / Un exemple : ISUP en vue de l'agrément}

On reprend les optimisations de l'agrément déjà mentionnées en 6/ et on reconstitue les classements des candidats (la totalité de la série de testage). Les éléments à prendre en considération dans l'ISUP sont l'ISU et deux coefficients de parenté : P1, parenté moyenne du candidat avec les autres candidats et P2, parenté moyenne du candidat avec toutes les femelles de la population. Les corrélations observées sur ces 3 variables et la variable de mérite sont indiquées au tableau 8 .

La corrélation entre le mérite et l'ISU est modérée et de l'ordre de 0,6 à 0,7 . La liaison entre le mérite et les coefficients de parenté est bien entendu négative (de l'ordre de - 0,4 à - 0,6). La liaison entre ISU et coefficients de parenté est positive, c'est-à-dire défavorable (de l'ordre de 0,2 ), sauf en race Holstein où elle est faiblement négative (favorable). A noter que sur des séries plus récentes, cette spécificité Holstein n'a pas été retrouvée : dans toutes les races, on a trouvé que la corrélation entre l'ISU et les coefficients de parenté était faiblement positive (de l'ordre de 0,1$)$. La liaison entre les deux critè-

Tableau 9. ISUP agrément: corrélations entre variables élémentaires.

\begin{tabular}{|c|c|c|c|c|}
\hline & Mérite & ISU & P1 & P2 \\
\hline \multirow{3}{*}{ Mérite } & $\mathrm{PH}$ & 0,74 & $-0,59$ & $-0,54$ \\
& $\mathrm{MO}$ & 0,56 & $-0,51$ & $-0,50$ \\
& $\mathrm{NO}$ & 0,59 & $-0,40$ & $-0,46$ \\
\hline \multirow{3}{*}{ ISU } & $\mathrm{PH}$ & & $-0,24$ & $-0,15$ \\
& $\mathrm{MO}$ & & 0,24 & 0,25 \\
& $\mathrm{NO}$ & & 0,28 & 0,24 \\
\hline \multirow{3}{*}{ P1 } & $\mathrm{PH}$ & & & 0,72 \\
& $\mathrm{MO}$ & & & 0,96 \\
& $\mathrm{NO}$ & & & 0,62 \\
\hline
\end{tabular}

race $\mathrm{PH}$ : Oger + Midatest ; race $\mathrm{MO}$ Umotest ; race NO : Gna. 
res de parenté est assez forte (supérieure à 0,6 ), particulièrement en race Montbéliarde. Il est à noter que l'ordre de grandeur de l'écart-type des coefficients de parenté exprimés en $\%$ est de 1 .

A partir de ces liaisons, l'ISUP correspond à la régression multiple du mérite prédit par l'ISU et les 2 coefficients de parenté. $\mathrm{Si}$ on donne par convention le poids 1 à l'ISU, les poids trouvés pour les parentés sont alors les suivants :

Tableau 10. Poids des parentés dans I'ISUP agrément.

\begin{tabular}{|l|c|c|}
\hline & P1 & P2 \\
\hline PH & -5 & -15 \\
\hline MO & -8 & -6 \\
\hline NO & -7 & -12 \\
\hline
\end{tabular}

race $\mathrm{PH}$ : Oger + Midatest ; race $\mathrm{MO}$ Umotest ; race NO : Gna.

Les coefficients de détermination de la prédiction sont de l'ordre de 0,80 $(0,82,0,76,0,81$ pour $\mathrm{PH}, \mathrm{MO}, \mathrm{NO}$ respectivement) ce qui veut dire que le mérite global est largement explicable à partir de variables simples. Cette prédiction est assez précise puisqu'environ $90 \%$ des taureaux agréés d'après la procédure complète d'optimisation l'auraient aussi été en se basant sur cet ISUP. Les coefficients de corrélation entre l'ISUP et les 3 variables de base sont alors les suivants :

Tableau 11. Corrélations entre ISUP agrément et variables de base.

\begin{tabular}{|l|c|c|c|}
\hline & ISU & P1 & P2 \\
\hline PH & 0,85 & $-0,68$ & $-0,62$ \\
\hline MO & 0,65 & $-0,58$ & $-0,57$ \\
\hline NO & 0,66 & $-0,45$ & $-0,51$ \\
\hline
\end{tabular}

race $\mathrm{PH}$ : Oger + Midatest ; race $\mathrm{MO}$

Umotest ; race NO : Gna.

Excepté pour le cas de la PH (spécifique à cette série comme mentionné plus haut), la corrélation entre l'ISU et l'ISUP est de l'ordre de 0,6-0,7. La coexistence de corrélations apparemment aussi peu élevées et de fortes différentielles de sélection pour l'ISU (cf les contraintes de calcul dans la phase d'optimisation) peuvent surprendre. Un élément d'explication réside dans le fait que les différentielles de sélection observées utilisées comme contraintes dans les optimisations a posteriori ne résultaient pas d'une sélection par troncature sur l'ISU.

\section{3 / Diffusion des ISUP}

On comprendra aisément que les coefficients des ISUP puissent varier suivant la structure généalogique momentanée de la population. Cette variation n'est pas une hypothèse gratuite car on a bien observé (données non montrées ici) que les éléments de base que sont les corrélations entre l'ISU et les parentés et entre parentés, peuvent varier suivant le Centre de Production de Semence (CPS) ou suivant l'année pour le même CPS. Par ailleurs, les coefficients de parenté attachés à un même individu sont susceptibles de varier dans le temps, en fonction de l'expansion ou de la raréfaction de certaines origines dans la population. Une publication officielle des ISUP à destination des éleveurs risquerait donc de les troubler, sans leur apporter de solutions pour la gestion quotidienne de leurs accouplements. L'ISUP est avant tout un outil de travail pour les unités de sélection.

\section{Discussion et conclusion}

L'examen de toutes les étapes de sélection dans les programmes concernant les bovins laitiers montre que l'utilisation de procédures optimisées aurait permis de diminuer d'environ $20 \%$ les coefficients de parenté moyens dans le groupe des reproducteurs, sans diminuer le niveau génétique pour l'index de synthèse, l'ISU. Ceci est vrai pour toutes les étapes et toutes les races considérées. On peut considérer que cette valeur représente une économie substantielle de variabilité génétique. Les conséquences d'une application réelle de ces procédures auraient été une diminution immédiate de la consanguinité, puis une remontée de celle-ci à un rythme environ $20 \%$ moins élevé qu'auparavant. Il est intéressant de signaler que cet écart d'environ $20 \%$ est retrouvé aussi dans les schémas de sélection porcins (Colleau et al 2004b, Colleau et Tribout 2006) et caprins (résultats non publiés). La constance de cette valeur indique que les comportements des sélectionneurs et des éleveurs sont en fait très voisins d'une espèce à l'autre.

On peut se réjouir d'une telle différence entre les schémas optimisés et les schémas réels, car cela permet d'entrevoir une possibilité d'amélioration pour ces derniers. On peut au contraire s'étonner qu'elle soit si importante, compte tenu du fait que les acteurs de la sélection sont conscients depuis longtemps du problème posé par la sauve- garde de la variabilité génétique. Les causes sont assurément multiples. D'abord, la sélection quantitative est effectuée non seulement d'après l'ISU mais d'après le détail des index élémentaires, ce qui diminue affaiblit d'autant la marge de manœuvre utilisable pour réduire la parenté. De toute évidence, ce facteur intervient beaucoup chez l'éleveur individuel et le mène à utiliser massivement les taureaux «complets» du moment. Par ailleurs, la perception du problème des parentés chez les sélectionneurs est incomplète, ce qui est normal vu la complexité du sujet : comme dit au début de ce texte, c'est la parenté moyenne dans le pool issu d'un brassage général entre sexes et entre classes d'âge qu'il faudrait apprécier correctement au moment de la prise de décision. On voit mal ici comment on pourrait se passer du calcul pour suppléer à l'intuition.

Les optimisations présentées ici ne sont que des rétrospectives. En raison des méthodes efficaces de calcul utilisées, elles sont dorénavant envisageables en mode prospectif, avec une certaine périodicité. Elles seront proposées aux sélectionneurs, pour préciser notamment les taux d'utilisation des taureaux d'IA impliqués dans les accouplements soit avec les mères à taureaux soit avec les vaches ordinaires, ce qui contribuerait à éclairer les décisions. La définition d'un ISU pénalisé pour la parenté est utile mais ne permet pas de faire l'économie de cette information, essentielle pour une gestion efficace de la variabilité génétique. Le conseil aux éleveurs en matière d'accouplements est extrêmement important si l'on désire que les efforts de gestion de la variabilité génétique en amont des programmes portent leurs fruits. Il doit non seulement satisfaire les exigences des éleveurs en matière de complémentarité entre vache et taureau mais aussi optimiser des critères généraux de la population, selon les concepts actuels de génétique quantitative.

\section{Remerciements}

Nous remercions particulièrement tous les professionnels responsables des programmes de sélection des bovins laitiers qui ont permis que leurs données soient utilisées en vue des tests rétrospectifs et accepté leur citation nominative. Les tout premiers tests ont été effectués en race Normande avec l'appui de J. Béchu, responsable du 
Gna. Ils ont été ensuite étendus à la race Holstein (Oger : J.A Coquereau) et à la race Montbéliarde (Umotest : M. Tissier et G. Mervant). L'analyse de la phase mise au testage a été effectuée avec la collaboration de S. Fritz (UNCEIA), responsable de la coordination du programme SAM, et celle de la gestion détaillée au niveau de l'éleveur, avec la collaboration de $\mathrm{H}$. de Préaumont (Isno).

\section{ANNEXE \\ Mode de calcul de la pondération parenté dans I'ISUP}

A l'issue des procédures d'optimisation, il est possible d'établir un classement des individus sans ambiguïté et sans ex aequo. D'abord, en tête de liste, les individus dont l'utilisation est recommandée, rangés par taux d'utilisation décroissante. Ensuite, les éliminés qu'on peut encore départager : en tête de liste, l'individu qui a été éliminé le dernier dans la procédure et en fin de liste, l'individu qui a été éliminé le premier.

Un retour à la procédure d'optimisation permet de comprendre comment ce classement est constitué. A la base, la procédure cherche à établir la fréquence optimale de tous les accouplements possibles entre candidats. Pour leur très grande majorité, la fréquence optimale est nulle, ce qui correspond à une non recommandation. A partir des fréquences trouvées pour les autres, on déduit les taux d'utilisation des reproducteurs mâles et femelles sélectionnés.

Les équations analytiques d'optimisation se présentent sous forme de systèmes linéaires avec autant d'inconnues que d'accouplements et avec des contraintes : la somme des fréquences doit être égale à 1 et la moyenne pondérée des valeurs génétiques ISU associées à chaque accouplement doit être égale à une valeur décidée à l'avance.

Les solutions fournies au début comportent des valeurs négatives, ce qui est choquant parce que toute fréquence doit être positive (entre 0 et 1). Heureusement, il est possible de démontrer que ces inconnues peuvent être définitivement considérées comme nulles. Les calculs sont alors recommencés itérativement jusqu'à ce qu'il n'y ait plus de valeurs négatives. On peut alors démontrer que les valeurs finales ne peuvent être supérieures à 1. Le problème d'optimisation est résolu.

II est possible de tenir la comptabilité des accouplements éliminés et de là, celle des animaux éliminés. Un groupe de candidats qui vient juste d'être éliminé est bien entendu plus «méritant» que les groupes déjà éliminés. Un classement interne dans ce groupe est ensuite effectué : en tête de liste, l'individu dont la contribution totale est la moins négative et en fin de liste, celui dont la contribution totale est la plus négative. Et ainsi de suite, jusqu'à ce qu'il n'y ait plus de candidats nouvellement éliminés.

On a donc à la fin un classement pour les candidats et un classement pour les candidates. Ces variables dépendent bien entendu de l'effectif total. C'est pourquoi il est nécessaire de les transformer pour s'affranchir de cet inconvénient. L'idée est de postuler l'existence d'un variable continue de «mérite» $(m)$ suivant une distribution normale (par exemple la distribution normale standard : moyenne 0 et écart-type 1). Elle n'est pas observée directement mais conditionne le classement. Pour un rang donné du classement, on considère alors l'espérance de la variable sous-jacente $(m)$. Soit par exemple à calculer le mérite d'un individu classé 10 ème dans une liste de 1000 individus. On effectue alors un grand nombre de répliques d'un tirage de 1000 valeurs indépendantes dans la distribution normale. Le mérite de l'individu est alors la moyenne sur toutes les répliques, de la 10ème plus grande valeur. Les plus méritants ont alors une valeur très positive (de l'ordre de +3 ) et les moins méritants une valeur très négative (de l'ordre de -3 ).

L'ISUP correspond à la prédiction de cette variable sous-jacente par régression linéaire intégrant les éléments facilement accessibles que sont l'ISU et certains critères de parentés. La pondération à accorder à la parenté découle alors directement des coefficients de régression trouvés pour la population considérée.

\section{Références}

Avendano S., Villanueva B., Woolliams J.A., 2003. Expected increases in genetic merit from using optimised contributions in two livestock populations of beef cattle and sheep. J. Anim. Sci., 79, 2964-2975.

Avendano S., Woolliams J.A., Villanueva B., 2004. Mendelian sampling terms as a selective advantage in optimum breeding schemes with restrictions on the rate of inbreeding. Genet. Res., 83, 55-64.

Ballou J.D., Lacy R.C., 1995. Identifying genetically important individuals for management of genetic variation in pedigreed populations. In : Ballou J.D., Gilpin M., Foose T.J., (Eds), Population management for survival and recovery, Columbia University press, New-York, 76-111.

Bijma P., Woolliams J.A., 1999. Prediction of genetic contributions and generation intervals in populations with overlapping generations under selection. Genetics, 151, 1197-1210.
Bijma P., Woolliams J.A., 2000. Predictions of rates of inbreeding in populations selected on best linear unbiased prediction of breeding value. Genetics, 156, 361-373.

Boichard D., Maignel L., Verrier E., 1996. Analyse généalogique des races bovines laitières françaises. INRA Prod. Anim., 9 (5), 323-335.

Boichard D., Maignel L., Verrier E., 1997. The value of using probabilities of gene origin to measure genetic variability in a population. Genet. Sel. Evol., 29, 5-23.

Brisbane J.R., Gibson J.P., 1995a. Balancing selection response and rate of inbreeding by including genetic relationships in selection decisions. Theor. Appl. Genet., 421-431.

Brisbane J.R., Gibson J.P., 1995b. Balancing selection response and rate of inbreeding by including predicted stabilised genetic contributions in selection decisions. Genet. Sel. Evol. 27, 541-549.
Caballero A.,1994. Developments in the prediction of effective population size. Heredity, 73, 657-679.

Caballero A., Santiago E., Toro M.A., 1996. Systems of mating to reduce inbreeding in selected populations. Anim. Sci., 431-442.

Caballero A., Toro M.A., 2000. Interrelations between effective population size and other pedigree tools for the management of conserved populations. Genet. Res., 75, 331-343.

Colleau J.J. 1999. Début de la sélection assistée par marqueurs chez les bovins laitiers : étude par simulation. Renc. Rech. Rum., 6, 231-233.

Colleau J.J., 2002. An indirect approach to the extensive calculation of relationship coefficients. Genet. Sel. Evol., 34, 409-421.

Colleau J.J., Regaldo D., 2001. Définition de l'objectif de sélection dans les races bovines laitières. Renc. Rech. Rum., 8, 329-332. 
Colleau J.J., Moureaux S., Briend M., Béchu J., 2003. Gestion dynamique de la variabilité génétique lors de la sélection des bovins laitiers. Renc. Rech. Rum., 10, 181- 184

Colleau J.J., Moureaux S., Briend M., Béchu J., 2004a. A method for the dynamic management of genetic variability in dairy cattle. Genet. Sel. Evol., 36, 373-394.

Colleau J.J., Moureaux S., Briend M., T. Tribout, 2004b. Management of selected populations: from theory to practice. $55^{\text {th }}$ EAAP Meeting, September 3-8 2004, Bled Slovenia. Commission on Animal Genetics, 11p.

Colleau J.J., Tribout T., 2006. Gestion optimisée de la variabilité génétique dans les populations porcines sélectionnées : exemple d'application sur la population collective Landrace Français Journ. Rech. Porcine en Fr., 38, 131136

Crow J.F., Kimura M., 1970. An introduction to population genetics theory. Burgess publishing company Minneapolis USA, 459p.

Falconer D.S., MacKay T.F.C., 1996. Introduction to quantitative genetics. $4^{\text {th }} \mathrm{Ed}$. Longman Sci. and Tech., Harlow UK, 464p.

Fritz S., Colleau J.J., Druet T., Boscher M.Y., Rossignol M.N., Malafosse A., Boichard D., 2003. Mise en place d'une sélection assistée par marqueurs dans les trois principales races bovines laitières françaises. Renc. Rech. Rum., 10, 53-57.

Grundy B. Hill W.G., 1993. A method for reducing inbreeding with best linear unbiased prediction. Anim. Prod., 56, 427-435.

Grundy B., Caballero A., Santiago E., Hill W.G., 1994. A note on using biased parameter values and non-random mating to reduce rates of inbreeding in selection programmes. Anim. Prod., 59, 465-468.

Hill W.G., 1972. Effective size of populations with overlapping generations. Theor. Pop. Biol., 3, 278-289.

Hill W.G., 1979. A note on effective population size with overlapping generations. Genetics, 92, 317-322.

Kearney J.F., Wall E., Villanueva B., Coffey M.P., 2004. Inbreeding trends and application of optimised selection in the UK Holstein population. J. Dairy Sci., 87, 3503-3509.

Lacy R., 1995. Clarification of genetic terms and their use in the management of captive populations. Zoo Biol., 14, 565-578.
Malécot G., 1948. Les mathématiques de l'hérédité. Masson et Cie, Paris, france, 63p.

Meuwissen T.H.E., 1997. Maximizing the response of selection with a predefined rate of inbreeding. J. Anim. Sci., 75, 934-940.

Meuwissen T.H.E., Sonesson A.K., 1998. Maximizing the response of selection with a predefined rate of inbreeding: overlapping generations. J. Anim. Sci., 76, 2575-2583.

Minvielle F., 1990. Principes d'amélioration génétique des animaux domestiques. Université Laval, Québec, et Institut National de la Recherche Agronomique, Paris, France, 211p.

Moureaux S., Boichard D., Verrier E., 2000. Utilisation de l'information généalogique pour l'estimation de la variabilité génétique de huit races bovines laitières française d'expression nationale ou régionale. Renc.Rech.Rum., 7, 149. 152.

Ollivier L., 2002. Eléments de génétique quantitative. $2^{\mathrm{e}}$ édition. INRA éditions. Paris, France, 184p.

Santiago E., Caballero A., 1995. Effective size of populations under selection. Genetics, 139, 1013-1030.

Sargolzaei M., Iwaisaki H., Colleau J.J., 2005a. A fast algorithm for computing inbreeding coefficients in large populations. J. Anim. Breed. Genet., 122, 325-331.

Sargolzaei M., Iwaisaki H., Colleau J.J., $2005 \mathrm{~b}$. Efficient computation of the inverse of gametic relationship matrix for a marked QTL. Genet. Sel. Evol,. (accepté).

Sonesson A.K., Meuwissen T.H.E., 2000. Mating schemes for optimum contribution selection with constrained rates of inbreeding. Genet. Sel. Evol., 32, 231-249.

Sonesson A.K., Grundy B., Woolliams J.A., Meuwissen THE. 2000. Selection with control of inbreeding in populations with overlapping generations: a comparison of methods. Anim. Sci., $70,1-8$.

Sonesson A.K., Meuwissen T.H.E., 2002. Non-random mating for selection with restricted rates of inbreeding and overlapping generations. Genet. Sel. Evol., 34, 23-39.

Toro M.A., Nieto B.M., 1984. A simple method for increasing the response to artificial selection. Genet. Res., 347-349.

Toro M.A., Perez-Enciso M., 1990. Optimisation of selection response under restricted inbreeding. Genet. Res. Evol., 22, 347-349.

Verrier E., Colleau J.J., Foulley J.L., 1993. Long-term effects of selection based on the animal model BLUP in a finite population. Theor. Appl. Genet., 87, 446-454.

Villanueva B., Woolliams J.A., Simm G., 1994. Strategies for controlling rates of inbreeding in MOET nucleus schemes for beef cattle. Genet. Sel. Evol., 26, 517-535.

Woolliams J.A., 1998. A recipe for the design of breeding schemes. In : Proceedings of the $6^{\text {th }}$ World Congress on Genetics Applied to Livestock Production, 11-16 January 1998, 25, University of New England, Armidale, Australia, 451-454.

Woolliams J.A., Wray N.R., Thompson R., 1993. Prediction of long-term contributions and inbreeding in populations undergoing mass selection. Genet. Res., 62, 231-242.

Woolliams J.A., Thompson R., 1994. A theory of genetic contributions. In Proceedings of the $5^{\text {th }}$ World Congress on Genetics Applied to Livestock Production, 19, 127-136

Woolliams J.A., Bijma P., 2000. Prediction of rates of inbreeding in populations undergoing selection. Genetics, 154, 1851-1864.

Woolliams J.A., Pong-Wong R., Villanueva B., 2002.Strategic optimisation of short-and long-term gain and inbreeding in MAS and nonMAS schemes. Session 23: Design of breeding programmes $7^{\text {th }}$ World Congress on Genetics Applied to Livestock Production August 19-23 2002, Montpellier, France. Communication 2302

Wray N.R., Thompson R., 1990. Prediction of rates of inbreeding in selected populations. Genet. Res., 55, 41-54.

Wray N.R., Woolliams J.A., Thompson R., 1994. Prediction of rates of inbreeding in populations undergoing index selection. Theor. Appl. Genet., 87,503-512

Wray N.R., Goddard M.E., 1994. Increasing long-term response to selection, Genet.Sel.Evol., $26,431-451$.

Wu L., Schaeffer L.R., 2000. Reducing the effect of parent averages from animal solutions in mixed model equations. J. Anim. Breed. Genet., 117, 361-374.

\section{Résumé}

On décrit l'approche adoptée pour gérer la variabilité génétique au moment de la procréation des jeunes taureaux, (phase amont), la mise en testage, de l'utilisation des taureaux de service (phase aval) et du choix des taureaux à l'issue du testage (phase agrément). L'objectif est de minimiser la parenté moyenne deux à deux dans la population existante augmentée des produits à naître des accouplements programmés, tout en fixant le niveau génétique moyen de ceux-ci à une valeur souhaitée. Des tests a posteriori en vraie grandeur sont effectués sur les races Holstein, Montbéliarde, Normande. Par rapport aux accouplements effectivement réalisés, l'optimisation permet de réduire les parentés moyennes de l'ordre de 20 à $30 \%$ (phases amont et aval), en gardant le niveau génétique moyen observé. L'agrément optimisé rejette une fraction importante des taureaux déjà agréés et par contre admet des taureaux qui n'ont pas reçu l'agrément. On analyse également les possibilités offertes par l'utilisation d'un index de synthèse pénalisé pour la parenté. 


\begin{abstract}
Optimizing management of kinship and inbreeding coefficients in dairy cattle selection

This paper describes the methodological approach followed for managing genetic variability in selected dairy cattle populations, when procreating young bulls (upward step), using service bulls (downward step) and selecting recently progeny-tested bulls (approval step). The objective was to minimize the average pairwise kinship coefficient in the existing population augmented by the individuals to be born from the planned matings, while setting their average genetic level to a pre-defined value. A posteriori full scale tests are carried out on the Holstein, Montbéliard, Norman breeds. In comparison with the most recent actual matings, the optimization method reduced the average kinship coefficients by about 20 to $30 \%$ (upward and downward steps), while maintaining the average genetic level to its observed value. Optimized approval rejects an important proportion of the bulls already approved and accepts some new bulls, previously dismissed. The potential of using an overall EBV penalized for coancestry was investigated.
\end{abstract}

COLLEAU J.-J., MOUREAUX S., 2006. Gestion optimisée de la parenté et de la consanguinité dans les programmes de sélection des bovins laitiers. INRA Prod. Anim., 19, 3-14. 
